\title{
铜催化二氟乙醇的芳基醚化反应及其机理研究
}

\author{
黄帅帅 ${ }^{a}$ 聂一雪 ${ }^{a}$ 杨晶晶 $^{a}$ 郑战江 ${ }^{*, a}$ 曹 建 ${ }^{a}$ \\ 徐 征 ${ }^{a}$ 徐利文*,a,b
}

( ${ }^{a}$ 杭州师范大学 有机硅化学及材料技术教育部重点实验室＼cjkstart杭州 311121)

$\left({ }^{b}\right.$ 杭州师范大学 氟硅精细化学品与材料制造协同创新中心 杭州 311121)

\begin{abstract}
摘要 有机氟硅化合物是元素有机化学中最重要的高端产品种类之一, 极具研究价值. 开发了含二氟乙醇农药的新工 艺技术，发展了一种条件温和和高效的铜催化二氟乙醇芳基醚化反应，通过大量的配体篮选和条件优化，建立了二氟 乙醇和芳基澳或芳基碘的 $\mathrm{C}-\mathrm{O}$ 偶联反应，简易合成出各种芳基取代的二氟乙基芳基醚. 该反应以 $\mathrm{CuI}$ 为催化剂，在 8羟基喹啉和叔丁醇钾存在下顺利进行, 具有广泛的底物适用性. ESI-MS 分析证明, 该催化过程可能存在三价铜催化机 制, 密度泛函理论(DFT)计算进一步表明该反应机理可能涉及三价铜的氧化加成、亲核取代及还原消除过程.
\end{abstract}

关键词 乌尔曼反应; 铜催化; 含氟化合物; DFT 计算; 8-羟基喹啉

\section{Copper-Catalyzed Arylated Etherification of 2,2-Difluoroethanol and Its Mechanistic Study}

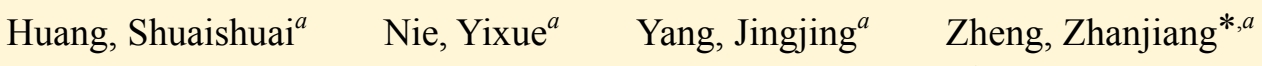 \\ Cao, $\operatorname{Jian}^{a} \quad \mathrm{Xu}$, Zheng $^{a} \quad \mathrm{Xu}$, Liwen ${ }^{*, a, b}$ \\ ( ${ }^{a}$ Key Laboratory of Organosilicon Chemistry and Material Technology of Ministry of Education, \\ Hangzhou Normal University, Hangzhou 311121) \\ ( ${ }^{b}$ Collaborative Innovation Center for Fluorosilicone-based Fine Chemicals and Materials, \\ Hangzhou Normal University, Hangzhou 311121)
}

\begin{abstract}
Both organofluorine and organosilicon compounds are one of the most important types of high-tech product in elementorganic chemistry, and have been received much attetions in the past decades. Considering the imporatance of difluoroethanol moeity in pesticides, the development of a mild and efficient copper-catalyzed arylated etherification reaction of difluoroethanol is highly desirable. Herein, a mild and efficient method for the preparation of difluoroethyl aryl ethers was developed by the copper-catalyzed Ullmann-type arylated etherification reaction of aryl bromides or iodides with 2,2-difluoroethanol. This reaction proceeds smoothly in the presence of $\mathrm{CuI}$ and 8-hydroxyquinoline/t-BuOK, and has a broad substrate scope. ESI-MS analysis supported the existence of $\mathrm{LCu}(\mathrm{III}) \operatorname{Ar}(\mathrm{OR})$ species during this catalytic reaction. Further density functional theory (DFT) calculations suggest a proposed mechanism of arylated etherification reaction involving oxidative addition, followed by nucleophile substitution and reductive elimination would be rational.

Keywords Ullmann reaction; Cu-catalyzed; fluorinated compound; DFT calculation; 8-hydroxyquinoline
\end{abstract}

\section{Introduction}

In the past decades, fluorine-containing compounds have been widely used in various fields such as material chemistry ${ }^{[1]}$ medicinal chemistry, ${ }^{[2]}$ and agrochemistry. ${ }^{[3]}$ The effect of incorporating one or several fluorine atoms into an organic substrate can have a vital effect on the physical, chemical, and biological properties of the molecule, which is exemplified by the growing number of pharmaceuticals and agrochemicals that contain fluorine atoms in their structure. ${ }^{[4]}$ Among organofluorinated molecules, difluoroethyl aryl ethers emerged as useful groups and were pre-

\footnotetext{
* Corresponding authors. E-mail: zzjiang78@hznu.edu.cn; liwenxu@hznu.edu.cn

Received March 14, 2020; revised April 17, 2020; published online April 23, 2020

Project supported by the National Natural Science Foundation of China (Nos. 21703051, 21801056) and the Hangzhou Science and Technology Bureau of China (No. 20180432B05)

国家自然科学基金(Nos. 21703051, 21801056)和杭州市科技局(No. 20180432B05)资助项目.
} 
sent in many bioactive substances due to high metabolic stability and significant lipophilicity of $\mathrm{CF}_{2} \mathrm{HCH}_{2} \mathrm{O}$ group (Scheme 1). ${ }^{[5,6]}$

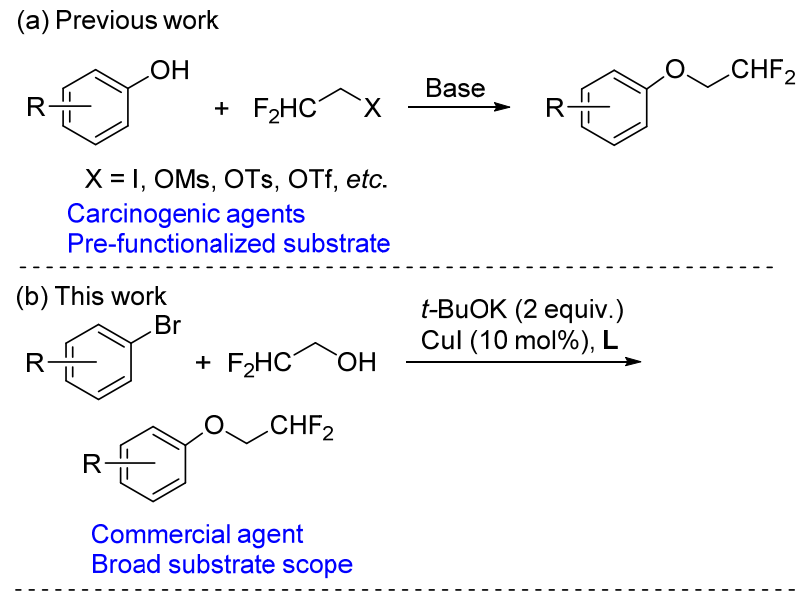

(c) Selected drug candidates or pesticide containing $\mathrm{OCH}_{2} \mathrm{CHF}_{2}$
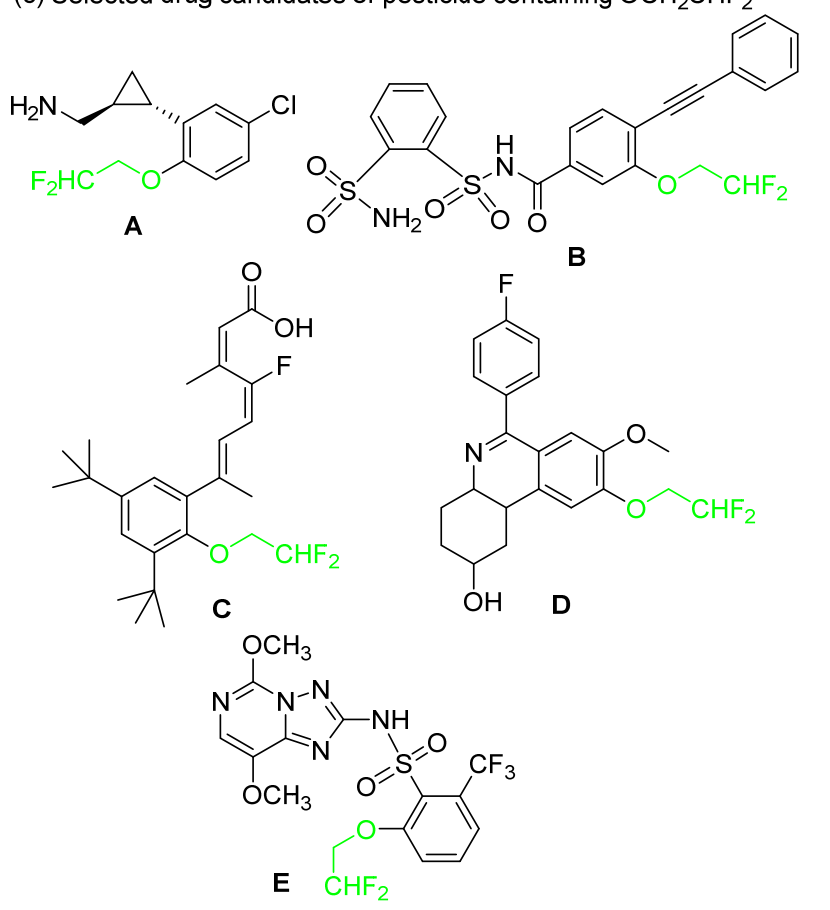

Scheme 1 Representative structures of bioactive molecules containing difluoroethyl aryl ether moiety

Meanwhile, methods have been reported over the past decades for the assembling of difluoroethyl aryl ethers. The main method relies on the Williamson ether synthesis, which involves the nucleophilic addition of a phenol with a difluoroethyl electrophile, ${ }^{[7]}$ such as difluoroethyl iodide or difluoroethyl mesylate. However, these strong alkylating agents are usually carcinogenic and therefore need extensive health and safety precautions (Scheme 1$).{ }^{[8]}$ Thus, the development of more general and applicable methods to prepare the difluoroethyl aryl ethers having been an important theme in organic chemistry and drug discovery. In this regard, palladium-catalyzed $\mathrm{C}-\mathrm{O}$ coupling reactions have been an efficient method over traditional methods. ${ }^{[9]}$ For example, Singh and coworkers ${ }^{[10]}$ reported a palladi- um-catalyzed cross-coupling reaction of primary fluoroalkyl alcohols with activated aryl halides. However, the limitation of this method is that these procedures required the use of the precious metal Pd and phosphine ligands. Inspired by the achievements of the $\mathrm{Cu}$-catalyzed $\mathrm{C}-\mathrm{O}$ coupling reaction, ${ }^{[11]}$ we assume that an alternative method that involves the copper-catalyzed arylated etherification reaction between aryl halide and commercial 2,2-difluoroethanol would be feasible (Scheme 1).

Generally speaking, traditional Ullmann-typed $\mathrm{C}-\mathrm{O}$ coupling procedures suffered from drawbacks such as harsh reaction conditions, stoichiometric amounts of copper and restricted range of substrates. ${ }^{[12]}$ In the past two decades, important breakthroughs were reported for copper-catalyzed $\mathrm{C}-\mathrm{O}$ coupling reaction with the use of appropriate ligands by the groups of Buchwald, Taillefer, Ma and Cai. ${ }^{[13]}$ Therefore, the methods for the coupling of aryl halides and 2,2,2-trifluoroethanol were also developed. ${ }^{[14]}$ However, there is no related report of the formation of difluoroethyl aryl ethers from 2,2-difluoroethanol directly. Probably due to the weaker nucleophilicity of the 2,2-difluoroethanol compared with the trifluoro one. Herein, we report the $\mathrm{CuI}$ catalyzed $\mathrm{C}-\mathrm{O}$ coupling-type arylated etherification reaction for the preparation of difluoroethyl aryl ethers.

\section{Results and discussion}

The reaction of 3-bromobenzotrifluoride (1a) and 2,2-difluoroethanol (2) in the presence of $\mathrm{K}_{3} \mathrm{PO}_{4}$ was initially examined, using $\mathrm{CuI}$ as the catalyst precursor and 8-hydroxyquinoline as the ligand at $100{ }^{\circ} \mathrm{C}$. However, no desired product was observed under this reported procedure (Table 1, Entry 1). ${ }^{[15]}$ Then the results of replacing $\mathrm{K}_{3} \mathrm{PO}_{4}$ with $\mathrm{Cs}_{2} \mathrm{CO}_{3}$ were checked. To our delight, the reaction proceeded well to give the desired product $3 \mathbf{a}$ in $75 \%$ GC yield (Table 1, Entry 2). Encouraged by this, further optimization was first done with a survey of the ligand. Unfortunately, it was found that when using 1,10-phenanthroline or ethyl 2-oxocyclohexanecarboxylate as ligand in the presence of $\mathrm{Cs}_{2} \mathrm{CO}_{3}$, the reaction proceeded sluggish to provide the target compound in trace yields (Table 1, Entries 3, 4). Given that the ligand/base can dramatically affect the yield, ${ }^{[16]}$ a thorough screening of a range of base with 8-hydroxyquinoline has been carried out (Table 1, Entries 5 14). It was found that when EtO$\mathrm{Na}, \mathrm{KOH}, \mathrm{NaHCO}_{3}, \mathrm{KHCO}_{3}, \mathrm{KF}, \mathrm{K}_{2} \mathrm{HPO}_{4}$, or $\mathrm{NaH}$ was used as base, only trace product was formed. When $\mathrm{NaOH}$ or $\mathrm{K}_{2} \mathrm{CO}_{3}$ was used, little or moderate product was obtained (Table 1, Entries 5, 13). Gratifyingly, the desired product 3a was achieved in quantitative GC yield when $t$-BuOK was used as base after $12 \mathrm{~h}$ in the presence of 8-hydroxyquinoline (Table 1, Entry 14). As expected, when $\mathrm{CF}_{3} \mathrm{CH}_{2} \mathrm{OH}$ was used instead of $\mathrm{CHF}_{2} \mathrm{CH}_{2} \mathrm{OH}$ in this condition, the reaction proceeded well in nearly quantitative GC yield. ${ }^{[14 \mathrm{~b}]}$

With the optimized reaction conditions in hand, the sub- 
Table 1 Optimization of reaction conditions ${ }^{a}$

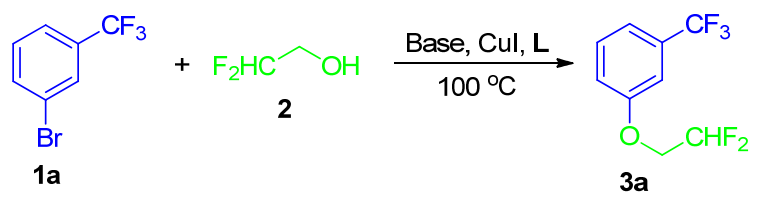

\begin{tabular}{cllc}
\hline Entry & \multicolumn{1}{c}{ Base } & \multicolumn{1}{c}{$\mathbf{L}$} & Yield ${ }^{b} \%$ \\
\hline 1 & $\mathrm{~K}_{3} \mathrm{PO}_{4}$ & 8-Hydroxyquinoline & 0 \\
2 & $\mathrm{Cs}_{2} \mathrm{CO}_{3}$ & 8-Hydroxyquinoline & 75 \\
3 & $\mathrm{Cs}_{2} \mathrm{CO}_{3}$ & 1,10-Phenanthroline & Trace \\
4 & $\mathrm{Cs}_{2} \mathrm{CO}_{3}$ & Ethyl 2-oxocyclohexane carboxylate & Trace \\
5 & $\mathrm{NaOH}^{2}$ & 8-Hydroxyquinoline & 13 \\
6 & $\mathrm{C}_{2} \mathrm{H}_{5} \mathrm{ONa}$ & 8-Hydroxyquinoline & Trace \\
7 & $\mathrm{KOH}$ & 8-Hydroxyquinoline & Trace \\
8 & $\mathrm{NaHCO}_{3}$ & 8-Hydroxyquinoline & Trace \\
9 & $\mathrm{~K}_{2} \mathrm{HPO}_{4}$ & 8-Hydroxyquinoline & Trace \\
10 & $\mathrm{KF}_{1}$ & 8-Hydroxyquinoline & Trace \\
11 & $\mathrm{KHCO}_{3}$ & 8-Hydroxyquinoline & Trace \\
12 & $\mathrm{NaH}_{13}$ & 8-Hydroxyquinoline & Trace \\
13 & $\mathrm{~K}_{2} \mathrm{CO}_{3}$ & 8-Hydroxyquinoline & 58 \\
14 & $t$ - $\mathrm{BuOK}^{2}$ & 8-Hydroxyquinoline & 100 \\
\hline
\end{tabular}

${ }^{a}$ Reaction conditions: 1a $(1.0 \mathrm{mmol}), 2(2 \mathrm{~mL})$, base (2 equiv.), CuI $(0.1$ equiv.) and ligand ( 0.2 equiv.), $100{ }^{\circ} \mathrm{C}$ for $12 \mathrm{~h} .{ }^{b} \mathrm{GC}$ yield.

strate generality of this copper-catalyzed arylated etherification of 2,2-difluoroethanol with various aryl halides was explored. As shown in Scheme 2, the reactions of aryl bromides containing $\mathrm{CF}_{3}$, methoxy or $\mathrm{Cl}$ group at me$t a$-positions provided the desired product in good isolated yields $(\mathbf{3 a} \sim \mathbf{3 c})$. Further, when aryl bromides bearing electron-withdrawing or electron-donating groups at $p a-$ $r a$-positions were used, the etherification reactions also proceeded smoothly to give the desired difluoroethyl aryl ethers in good yields $(\mathbf{3 d} \sim \mathbf{3 1})$. It is noted that when the aryl bromides containing two substituents at the 3,4 or 3,5 positions were used, the etherification reactions proceeded well with moderate to good yields $(\mathbf{3 m} \sim \mathbf{3 q})$. Particularly, tri-substituted 3,4,5-trimethoxybromobenzene was compatible with this method to furnish the corresponding ether with moderate yield (3r). In addition, 3-bromopyridine was well tolerated and the desired product (3v) was given in $73 \%$ isolated yield. However, the reactions of aryl bromides with orth-substituents gave the difluoroethyl aryl ethers in low yields owing to the steric effect $(\mathbf{3 s} \sim 3 \mathbf{3})$.

Next, the scope of aryl iodides was investigated for the $\mathrm{CuI}$ catalyzed $\mathrm{C}-\mathrm{O}$ coupling in the presence of 8 -hydroxyquinoline/t-BuOK with 2,2-difluoroethanol (Scheme 3). In general, various aryl iodides are suitable for this reaction. The steric and electronic effects of the substituents on yields are similar to aryl bromides. For example, aryl iodides with $\mathrm{NO}_{2}, \mathrm{CN}$ at para-position or $\mathrm{OMe}, \mathrm{Cl}, \mathrm{NO}_{2}$, $\mathrm{CF}_{3}$ at meta-positon, were all smoothly coupled with

Table $2 \mathrm{CuI} / 8$-Hydroxyquinoine-catalyzed arylated etherification reaction of aryl bromides with 2,2-difluoroethanol

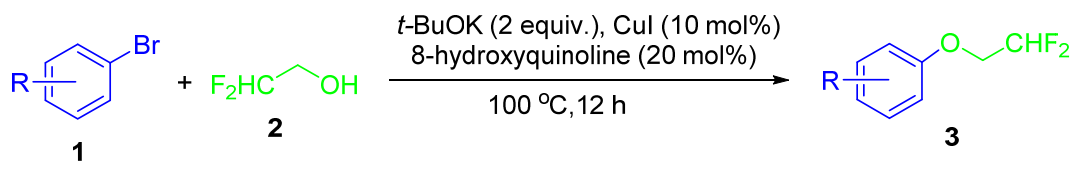<smiles>FC(F)COc1cccc(C(F)(F)F)c1</smiles>

3a, $75 \%$ yield<smiles>Fc1ccc(OCC(F)F)cc1</smiles>

3f, $82 \%$ yield<smiles>COc1ccc(OCC(F)F)cc1</smiles>

3k, $64 \%$ yield<smiles>CC(C)(C)c1cc(OCC(F)F)cc(C(C)(C)C)c1</smiles>

3 p, $57 \%$ yield<smiles>COc1cccc(OCC(F)F)c1</smiles>

3b, $67 \%$ yield<smiles>FC(F)COc1ccc(-c2ccccc2)cc1</smiles>

3g, $75 \%$ yield<smiles>CN(C)c1ccc(OCC(F)F)cc1</smiles>

3I, $76 \%$ yield<smiles>FC(F)COc1cc(C(F)(F)F)cc(C(F)(F)C(F)(F)F)c1</smiles>

$3 q, 45 \%$ yield<smiles>Fc1ccccc1OCC(F)F</smiles>

$3 u, 68 \%$ yield<smiles>FC(F)COc1cccc(Cl)c1</smiles>

3c, $66 \%$ yield<smiles>CC(C)(C)c1ccc(OCC(F)F)cc1</smiles>

3h, $76 \%$ yield<smiles>COc1ccc(OCC(F)F)cc1OC</smiles>

$3 \mathrm{~m}, 81 \%$ yield<smiles>COc1cc(OCC(F)F)cc(OC)c1OC</smiles>

$3 r, 54 \%$ yield<smiles>FC(F)COc1cccnc1</smiles>

$3 v, 73 \%$ yield<smiles>Cc1ccc(OCC(F)F)cc1</smiles>

3d, $64 \%$ yield<smiles>N#Cc1ccc(OCC(F)F)cc1</smiles>

$3 \mathbf{3 i}, 69 \%$ yield<smiles>Cc1cc(C)cc(OCC(F)F)c1</smiles>

$3 n, 78 \%$ yield<smiles>CC(=O)c1ccccc1OCC(F)F</smiles>

3 s, $33 \%$ yield<smiles>CC(=O)c1ccc(OCC(F)F)cc1</smiles>

3e, $75 \%$ yield<smiles>FC(F)COc1ccc(C(F)(F)F)cc1</smiles>

$3 j, 58 \%$ yield<smiles>COc1cc(OC)cc(OCC(F)F)c1</smiles>

3o, $71 \%$ yield $\mathrm{Ph}$<smiles>FC(F)COc1ccccc1-c1ccccc1</smiles>

3t, $12 \%$ yield 
Table $3 \mathrm{CuI} / 8$-Hydroxyquinoine catalyzed coupling reaction of aryl iodides with 2,2-difluoroethanol

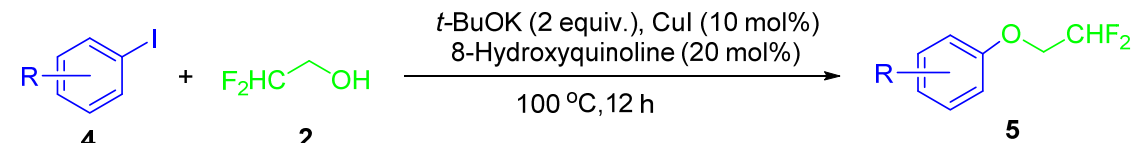

42

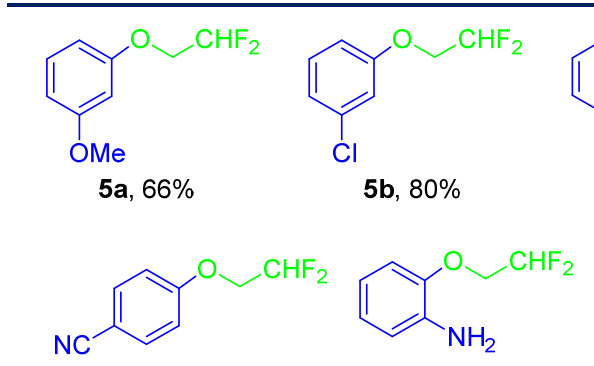

$5 \mathrm{~g}, 82 \%$

$5 \mathrm{~h}, 23 \%$

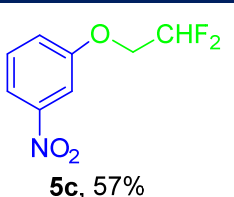

5c, $57 \%$

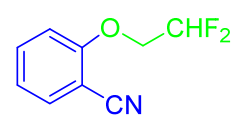

$5 \mathbf{i}, 53 \%$

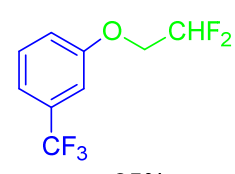

5d, $85 \%$<smiles>FC(F)COc1ccc(-c2ccccc2)cc1</smiles>

$5 e, 59 \%$

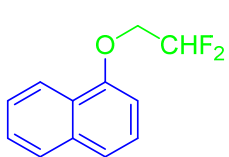

$\mathbf{5 k}, 10 \%$<smiles>O=[N+]([O-])c1ccc(OCC(F)F)cc1</smiles>

5f, $85 \%$

5j, $57 \%$

5I, $49 \%$

2,2-difluoroethanol, giving desired products in excellent yields $(\mathbf{5 a} \sim \mathbf{5 g})$. While aryl iodides with $\mathrm{NH}_{2}$ or $\mathrm{CN}$ at ortho-position only showed low reactivity with low to moderate yields $(\mathbf{5 h} \sim \mathbf{5 k})$. Though aryl iodides with $\mathrm{NH}_{2}$ or $\mathrm{CN}$ at ortho-position only showed low reactivity $(\mathbf{5 h} \sim$ $\mathbf{5 k}$ ), the corresponding products are valuable substrates for the hydrogen-borrowing reaction. ${ }^{[17]}$

Finally, we focused on elucidating the possible mechanism of this type of copper-catalyzed $\mathrm{C}-\mathrm{O}$ cross coupling reaction. Based on previous reports, most studies support the initial formation of $\mathrm{LCu}(\mathrm{I}) \mathrm{Nu}$ complex, then oxidative addition of aryl halide ( $\mathrm{ArBr}$ ) to the complex, followed by dissociation of $\mathrm{HBr}$ providing $\mathrm{LCu}(\mathrm{III}) \mathrm{Ar}(\mathrm{OR})$, which regenerates the active $\mathrm{Cu}(\mathrm{I})$ species and the desired product ArOR through reductive elimination. However, the detection of organometallic copper(III) complexes has been very scarce under this catalytic Ullmann reaction conditions. ${ }^{[18]}$ In order to have a better insight of the reaction pathway, several mechanistic experiments were carried out. Firstly, when a stoichiometric amount of radical scavenger 2,2,6,6tetramethylpiperidine-1-oxyl (TEMPO) was added, the reaction was not suppressed and the difluoroethyl aryl ether 3a was delivered in $97 \%$ GC yield, which indicated that the reaction probably didn't involve a radical process.
Further, in situ ESI-MS analysis was carried out. A solution of 2,2-difluoroethanol (5 equiv.), potassium tertbutoxide ( 2 equiv.), $m$-bromobenzotrifluo- ride ( 1 equiv.), $\mathrm{CuI}(10 \mathrm{~mol} \%)$ and 8 -hydroxyquinoline $(20 \mathrm{~mol} \%)$ was stirred at room temperature for $40 \mathrm{~min}$. ESI-MS analysis (Figure 1) of the reaction solution showed the peak $\mathrm{m} / \mathrm{z}$ 389.98 which is identified as $\left[\mathrm{CuL}_{2}\right]^{-} \mathrm{K}^{+}$and the peak of its dimer at $\mathrm{m} / \mathrm{z}$ 743.01. Very fortunately, the peak at $\mathrm{m} / \mathrm{z}$ 868.91 was observed, which is identified as the dimer of $\mathrm{LCu}(\mathrm{III}) \operatorname{Ar}(\mathrm{OR})$. Obviously, it is the direct evidence for the existence of $\mathrm{Cu}(\mathrm{III})$ intermediate during this difluoroethoxylation reaction.

On the basis of above experimental results and the literature precedence as well as our density functional theory (DFT) calculations, ${ }^{[19]}$ two plausible reaction pathways were proposed for this catalytic Ullmann-type arylated etherification reaction (Scheme 2). The path $\mathrm{A}$ begins with the coordination of the nucleophile $\mathrm{CHF}_{2} \mathrm{CH}_{2} \mathrm{O}^{-}$to the $\mathrm{Cu}(\mathrm{I})$ center $\left(\left[\mathrm{CuL}_{2}\right]^{-}\right)$to give $[\mathrm{LCu}-\mathrm{Nu}]^{-}$(Int 1), which is followed by the oxidative addition with aryl bromide to form the $\mathrm{LCu}(\mathrm{III}) \operatorname{Ar}(\mathrm{OR})$ species (Int 3). The subsequent reductive elimination gives the desired coupling product, together with the releasing of the $\mathrm{Cu}(\mathrm{I})$ species. The path $\mathrm{B}$ would start with the oxidative addition of the $\left[\mathrm{CuL}_{2}\right]^{-}$

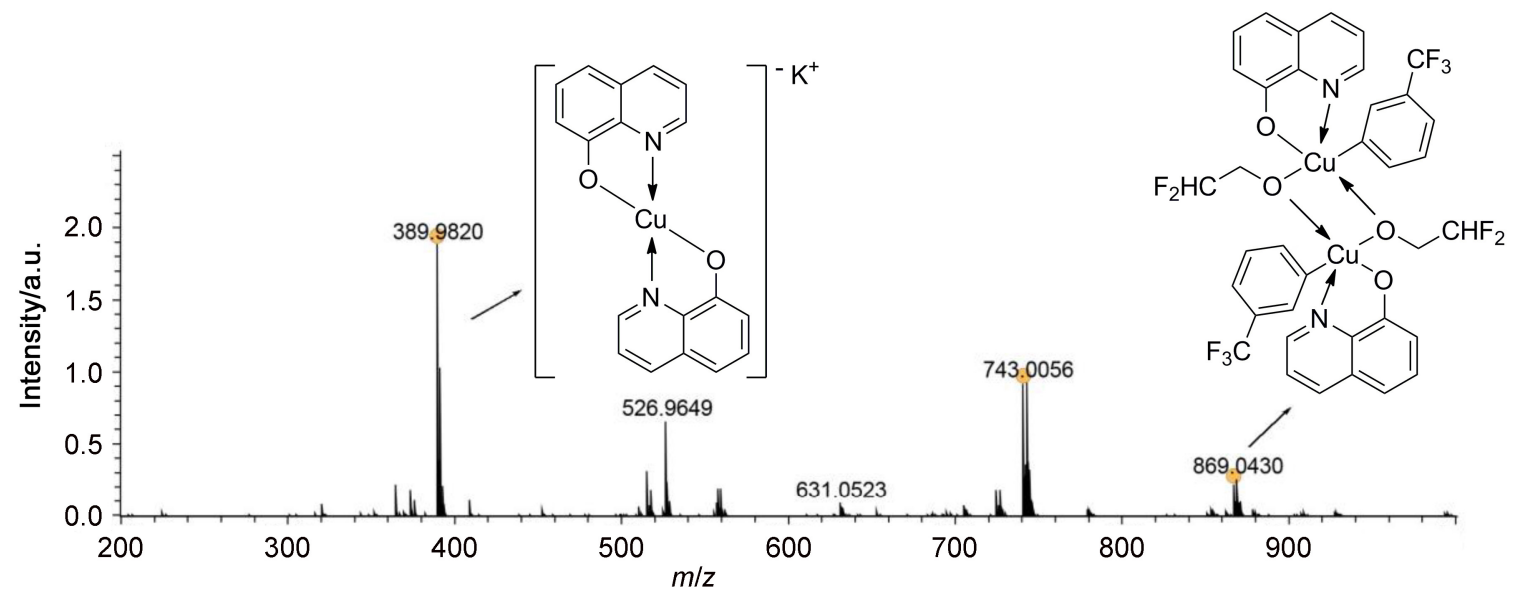

Figure 1 ESI-MS experiment on the CuI-catalyzed arylated etherification reaction between $m$-bromobenzotrifluoride and 2,2difluoroethanol 


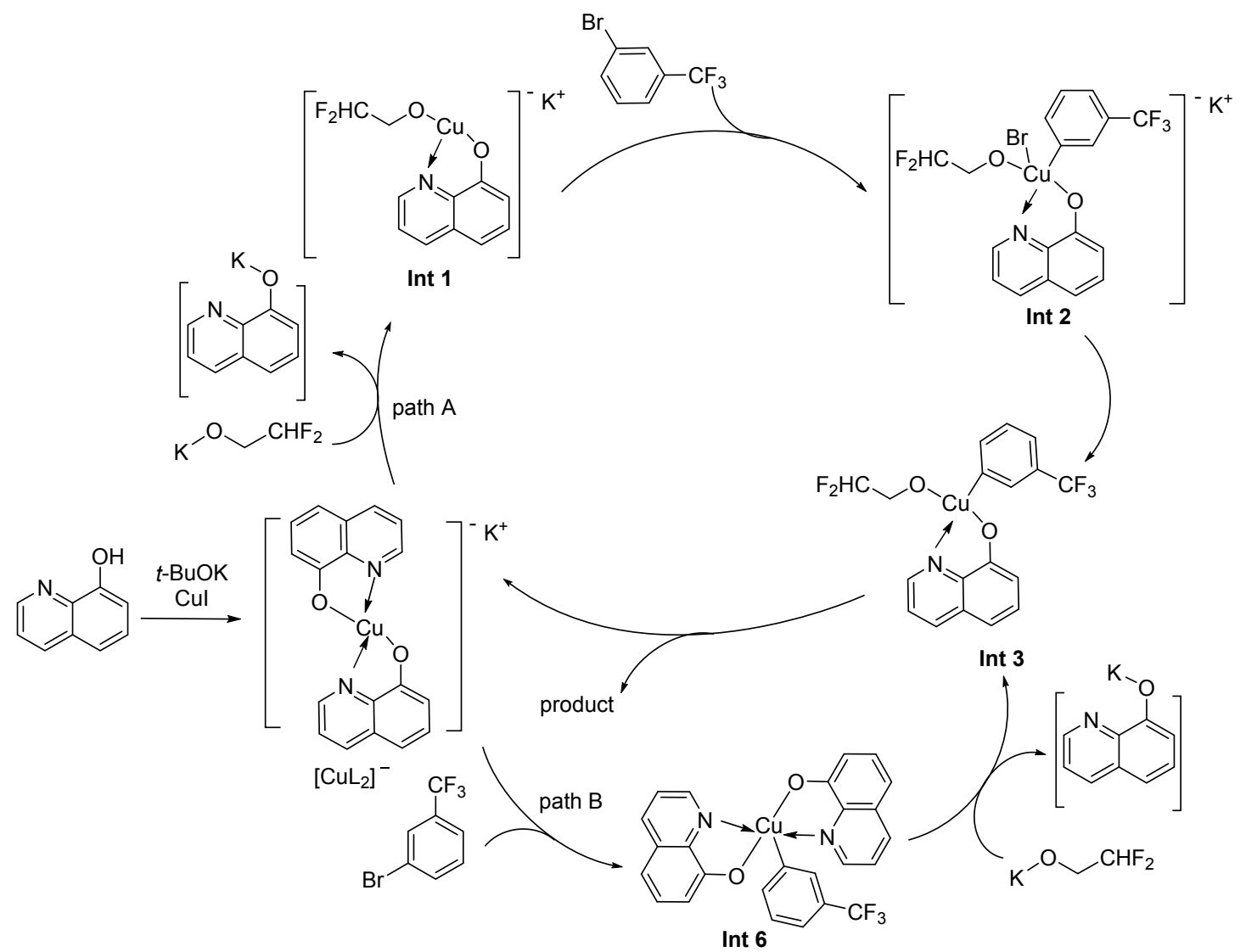

Scheme 2 Two proposed pathways for the Cu catalyzed reaction of aryl bromide (1a) and 2,2-difluoroethanol (2)

intermediate with aryl bromide, then the rapid ligand substitution with nucleophile forms the detectable $\mathrm{LCu}$ (III)$\operatorname{Ar}(\mathrm{OR})$ (Int 3). Finally, reductive elimination from Int 3 would then produce the coupling product. As shown in Figure S2 and Figure S3 (see supporting information), the two $\mathrm{Cu}(\mathrm{I})$ complexes $[\mathrm{LCu}-\mathrm{Nu}]^{-}$and $\left[\mathrm{CuL}_{2}\right]^{-}$would perform the oxidative addition with aryl bromide as the rate-limiting step of the reaction. However, the free energy barrier for oxidative addition of $[\mathrm{LCu}-\mathrm{Nu}]^{-}$in path $\mathrm{A}$ is $89.6 \mathrm{~kJ} / \mathrm{mol}$, while the free energy barrier of $\left[\mathrm{CuL}_{2}\right]^{-}$in path $\mathrm{B}$ is only $70.3 \mathrm{~kJ} / \mathrm{mol}$. It was due to that electron- rich $\left[\mathrm{CuL}_{2}\right]^{-}$would be more reactive toward oxidative addition than $[\mathrm{LCu}-\mathrm{Nu}]^{-}$complex. By comparison, the following reductive elimination is a facile step for both pathway with an energy barrier of $15.9 \mathrm{~kJ} / \mathrm{mol}$. Hence, the previous report suggested that the coordination of nucleophile to $\mathrm{Cu}(\mathrm{I})$ may occur before activation of the aryl halide (path A). In our case, however, the path B involving oxidative addition/nucleophile substitution and reductive elimination, is more reasonable.

\section{Conclusions}

In summary, we have developed a general and efficient method for the construction of difluoroethyl aryl ethers through the $\mathrm{C}-\mathrm{O}$ coupling-type arylated etherification reaction of aryl bromides or iodides with 2,2-difluoroethanol. The reaction proceeded smoothly with $\mathrm{CuI}$ as a catalyst and 8-hydroxyquinoline as a ligand in the presence of $t$-BuOK under mild conditions. In addition, ESI-MS studies reveal that $\mathrm{LCu}(\mathrm{III}) \mathrm{Ar}(\mathrm{OR})$ complex is involved in the reaction, and further DFT calculations support the new pathway of this $\mathrm{C}-\mathrm{O}$ coupling reaction.

\section{Experimental section}

\subsection{General information}

Unless specifically stated, all reagents were commercially obtained and purified prior to use if appropriate. Flash column chromatography was performed over silica (100 200 mesh). ${ }^{1} \mathrm{H}$ NMR and ${ }^{13} \mathrm{C}$ NMR spectra were recorded at 400 and $100 \mathrm{MHz}$, respectively on an Advance (Bruker) $400 \mathrm{MHz}$ Nuclear Magnetic Resonance Spectromer, and were referenced to the internal solvent signals. EI and CI mass spectra were performed on a Trace DSQ GC/ MS spectrometer. High Resolution Mass Spectra (ESIHRMS) were operated on a microOTOF-QII (Bruker). Thin layer chromatography was performed using Silica.

\subsection{General procedure for the coupling reaction of aryl bromides or aryl iodides with 2,2-difluoroethanol}

To a solution of $\mathrm{CuI}$ (19 mg, $0.1 \mathrm{mmol})$, 8-hydroxyquinoline (29 $\mathrm{mg}, 0.2 \mathrm{mmol})$ and $t$-BuOK (224 mg, 2 $\mathrm{mmol})$ in 2,2-difluoroethanol $(2 \mathrm{~mL})$ was added aryl bromide or aryl iodides $(1 \mathrm{mmol})$. The mixture was heated at $100{ }^{\circ} \mathrm{C}$ for $12 \mathrm{~h}$ and then saturated $\mathrm{NH}_{4} \mathrm{Cl}(30 \mathrm{~mL})$ was added. The mixture was filtered through a pad of celite, and extracted with EtOAc $(30 \mathrm{~mL} \times 3)$. The combined or- 
ganic phase was dried over $\mathrm{Na}_{2} \mathrm{SO}_{4}$, concentrated in vacuo, and purified by flash column chromatograph on silica gel to give the corresponding coupling product.

1-(2,2-Difluoroethoxy)-3-(trifluoromethyl)benzene (3a): Light yellow liquid. ${ }^{1} \mathrm{H}$ NMR $\left(400 \mathrm{MHz}, \mathrm{CDCl}_{3}\right) \delta: 7.43(\mathrm{t}$, $J=8.0 \mathrm{~Hz}, 1 \mathrm{H}), 7.31 \sim 7.25(\mathrm{~m}, 1 \mathrm{H}), 7.16(\mathrm{~s}, 1 \mathrm{H}), 7.10$ (dd, $J=8.4,2.0 \mathrm{~Hz}, 1 \mathrm{H}), 6.11(\mathrm{tt}, J=54.8,4.0 \mathrm{~Hz}, 1 \mathrm{H})$, $4.22(\mathrm{td}, J=12.8,4.0 \mathrm{~Hz}, 2 \mathrm{H}) ;{ }^{13} \mathrm{C}$ NMR $(100 \mathrm{MHz}$, $\left.\mathrm{CDCl}_{3}\right) \delta: 158.0,132.3(\mathrm{q}, J=33 \mathrm{~Hz}), 130.4,123.9$ (q, $J=$ $267 \mathrm{~Hz}), 118.9$ (q, $J=4 \mathrm{~Hz}), 118.2,113.5$ (t, $J=240 \mathrm{~Hz})$, 111.7 (q, $J=4 \mathrm{~Hz}), 67.6$ (t, $J=30 \mathrm{~Hz}$ ); HRMS (APCI) calcd for $\mathrm{C}_{9} \mathrm{H}_{7} \mathrm{~F}_{5} \mathrm{O}: 226.0412$, found 226.0420 .

1-(2,2-Difluoroethoxy)-3-methoxybenzene (3b): Colorless liquid. ${ }^{1} \mathrm{H}$ NMR $\left(400 \mathrm{MHz}, \mathrm{CDCl}_{3}\right) \delta: 7.20$ (t, $J=8.1$ $\mathrm{Hz}, 1 \mathrm{H}), 6.62 \sim 6.54(\mathrm{~m}, 1 \mathrm{H}), 6.54 \sim 6.44(\mathrm{~m}, 2 \mathrm{H}), 6.07(\mathrm{tt}$, $J=55.2,4.4 \mathrm{~Hz}, 1 \mathrm{H}), 4.16(\mathrm{td}, J=13.2,4.4 \mathrm{~Hz}, 2 \mathrm{H}), 3.79$ $(\mathrm{s}, 3 \mathrm{H}) ;{ }^{13} \mathrm{C}$ NMR $\left(100 \mathrm{MHz}, \mathrm{CDCl}_{3}\right) \delta: 161.1,159.1$, 130.3, $113.8(\mathrm{t}, J=240 \mathrm{~Hz}), 107.7,106.6,101.5,67.4(\mathrm{t}$, $J=30 \mathrm{~Hz}$ ), 55.5; HRMS (APCI) calcd for $\mathrm{C}_{9} \mathrm{H}_{11} \mathrm{~F}_{2} \mathrm{O}_{2}[\mathrm{M}+$ $\mathrm{H}]^{+}:$189.0722, found 189.0726 .

1-Chloro-3-(2,2-difluoroethoxy)benzene (3c): Colorless liquid. ${ }^{1} \mathrm{H}$ NMR $\left(400 \mathrm{MHz}, \mathrm{CDCl}_{3}\right) \delta: 7.23$ (dd, $J=10.4$, $2.4 \mathrm{~Hz}, 1 \mathrm{H}), 7.03 \sim 6.98(\mathrm{~m}, 1 \mathrm{H}), 6.95 \sim 6.90(\mathrm{~m}, 1 \mathrm{H})$, $6.84 \sim 6.79(\mathrm{~m}, 1 \mathrm{H}), 6.08(\mathrm{tt}, J=54.8,4.0 \mathrm{~Hz}, 1 \mathrm{H}), 4.16$ $(\mathrm{td}, J=13.2,4.0 \mathrm{~Hz}, 2 \mathrm{H}) ;{ }^{13} \mathrm{C}$ NMR $\left(100 \mathrm{MHz}, \mathrm{CDCl}_{3}\right) \delta$ : $158.5,135.3,130.6,130.4,122.4,115.3,113.6(\mathrm{t}, J=240$ $\mathrm{Hz}), 113.1,67.5$ (t, $J=30 \mathrm{~Hz}$ ); HRMS (APCI) calcd for $\mathrm{C}_{8} \mathrm{H}_{7} \mathrm{ClF}_{2} \mathrm{O}$ : 192.0148, found 192.0152.

1-(2,2-Difluoroethoxy)-4-methylbenzene (3d): Colorless liquid. ${ }^{1} \mathrm{H} \mathrm{NMR}\left(400 \mathrm{MHz}, \mathrm{CDCl}_{3}\right) \delta: 7.02(\mathrm{~d}, J=8.3 \mathrm{~Hz}$, $2 \mathrm{H}), 6.73(\mathrm{~d}, J=8.6 \mathrm{~Hz}, 2 \mathrm{H}), 5.98(\mathrm{tt}, J=55.2,4.0 \mathrm{~Hz}$, $1 \mathrm{H}), 4.06(\mathrm{td}, J=13.2,4.0 \mathrm{~Hz}, 2 \mathrm{H}), 2.23(\mathrm{~s}, J=9.6 \mathrm{~Hz}$, $3 \mathrm{H}) ;{ }^{13} \mathrm{C}$ NMR (100 MHz, $\left.\mathrm{CDCl}_{3}\right) \delta: 155.8,131.4,130.2$, 114.7, 114.0 (t, $J=240 \mathrm{~Hz}), 67.6(\mathrm{t}, J=30 \mathrm{~Hz}), 20.6$; HRMS (APCI) calcd for $\mathrm{C}_{9} \mathrm{H}_{11} \mathrm{~F}_{2} \mathrm{O}[\mathrm{M}+\mathrm{H}]^{+}:$173.0772, found 173.0779 .

1-(2-(2,2-Difluoroethoxy)phenyl)ethanone (3e): Pale yellow solid, m.p. $48 \sim 50{ }^{\circ} \mathrm{C} ;{ }^{1} \mathrm{H}$ NMR $(400 \mathrm{MHz}$, $\left.\mathrm{CDCl}_{3}\right) \delta: 7.99 \sim 7.93(\mathrm{~m}, 2 \mathrm{H}), 6.96(\mathrm{~d}, J=8.9 \mathrm{~Hz}, 2 \mathrm{H})$, $6.12(\mathrm{tt}, J=54.8,4.0 \mathrm{~Hz}, 1 \mathrm{H}), 4.25(\mathrm{td}, J=12.8,4.0 \mathrm{~Hz}$, 2H), $2.57(\mathrm{~s}, 3 \mathrm{H}) ;{ }^{13} \mathrm{C}$ NMR $\left(100 \mathrm{MHz}, \mathrm{CDCl}_{3}\right) \delta: 196.7$, $161.5,131.6,130.8,114.4,113.5(\mathrm{t}, J=240 \mathrm{~Hz}), 67.3(\mathrm{t}$, $J=30 \mathrm{~Hz}$ ), 26.5; HRMS (APCI) calcd for $\mathrm{C}_{10} \mathrm{H}_{11} \mathrm{~F}_{2} \mathrm{O}_{2}$ $[\mathrm{M}+\mathrm{H}]^{+}:$201.0722, found 201.0730.

1-(2,2-Difluoroethoxy)-4-fluorobenzene (3f): Colorless liquid. ${ }^{1} \mathrm{H} \mathrm{NMR}\left(400 \mathrm{MHz}, \mathrm{CDCl}_{3}\right) \delta: 7.03 \sim 6.94(\mathrm{~m}, 2 \mathrm{H})$, $6.90 \sim 6.82(\mathrm{~m}, 2 \mathrm{H}), 6.07(\mathrm{tt}, J=55.2,4.0 \mathrm{~Hz}, 1 \mathrm{H}), 4.14$ $(\mathrm{td}, J=12.8,4.0 \mathrm{~Hz}, 2 \mathrm{H}) ;{ }^{13} \mathrm{C} \mathrm{NMR}\left(100 \mathrm{MHz}, \mathrm{CDCl}_{3}\right) \delta$ : $159.3,156.9,154.0,116.3(\mathrm{~d}, J=20 \mathrm{~Hz}), 116.0$ (d, $J=8$ $\mathrm{Hz}), 116.0,113.8(\mathrm{t}, J=240 \mathrm{~Hz}), 68.2(\mathrm{t}, J=29 \mathrm{~Hz})$; HRMS (APCI) calcd for $\mathrm{C}_{8} \mathrm{H}_{7} \mathrm{~F}_{3} \mathrm{O}:$ 176.0444, found 176.0447 .

4-(2,2-Difluoroethoxy)biphenyl (3g): White solid, m.p. $87 \sim 89{ }^{\circ} \mathrm{C} ;{ }^{1} \mathrm{H}$ NMR (400 MHz, $\left.\mathrm{CDCl}_{3}\right) \delta: 7.57 \sim 7.51$ (m, 4H), $7.42(\mathrm{t}, J=7.6 \mathrm{~Hz}, 2 \mathrm{H}), 7.34(\mathrm{~s}, 1 \mathrm{H}), 7.32(\mathrm{t}, J=$ $7.3 \mathrm{~Hz}, 1 \mathrm{H}), 6.11(\mathrm{tt}, J=54.8,4.0 \mathrm{~Hz}, 1 \mathrm{H}), 4.22(\mathrm{td}, J=$ $12.8,4.0 \mathrm{~Hz}, 2 \mathrm{H}) ;{ }^{13} \mathrm{C} \mathrm{NMR}\left(100 \mathrm{MHz}, \mathrm{CDCl}_{3}\right) \delta: 157.4$, $140.6,135.3,128.9,128.5,127.1,127.0,115.1,113.9(\mathrm{t}$, $J=240 \mathrm{~Hz}), 67.57(\mathrm{t}, J=30 \mathrm{~Hz}$ ); MS (EI) $m / z: 234,215$, $183,169,153,76$.

1-tert-Butyl-4-(2,2-Difluoroethoxy)benzene(3h): Colorless liquid. ${ }^{1} \mathrm{H}$ NMR $\left(400 \mathrm{MHz}, \mathrm{CDCl}_{3}\right) \delta: 7.24$ (d, $J=8.8$ $\mathrm{Hz}, 2 \mathrm{H}), 6.77$ (d, $J=8.8 \mathrm{~Hz}, 2 \mathrm{H}), 5.99(\mathrm{tt}, J=55.6,4.4 \mathrm{~Hz}$, $1 \mathrm{H}), 4.08(\mathrm{td}, J=13.2,4.0 \mathrm{~Hz}, 2 \mathrm{H}), 1.22(\mathrm{~s}, 9 \mathrm{H}) ;{ }^{13} \mathrm{C} \mathrm{NMR}$ $\left(100 \mathrm{MHz}, \mathrm{CDCl}_{3}\right) \delta: 155.7,144.9,126.6,114.3,114.0(\mathrm{t}$, $J=240 \mathrm{~Hz}$ ), $67.6(\mathrm{t}, J=30 \mathrm{~Hz}), 34.3,31.6$; HRMS (APCI) calcd for $\mathrm{C}_{12} \mathrm{H}_{16} \mathrm{~F}_{2} \mathrm{O}: 214.1164$, found 214.1150.

4-(2,2-Difluoroethoxy)benzonitrile (3i): Colorless liquid. ${ }^{1} \mathrm{H}$ NMR $\left(400 \mathrm{MHz}, \mathrm{CDCl}_{3}\right) \delta: 7.63(\mathrm{~d}, J=8.9 \mathrm{~Hz}$, 2H), 6.99 (d, $J=8.9 \mathrm{~Hz}, 2 \mathrm{H}), 6.11(\mathrm{tt}, J=54.8,4.0 \mathrm{~Hz}$, $1 \mathrm{H}), 4.24$ (td, $J=12.8,4.0 \mathrm{~Hz}, 2 \mathrm{H}) ;{ }^{13} \mathrm{C} \mathrm{NMR}(100 \mathrm{MHz}$, $\left.\mathrm{CDCl}_{3}\right) \delta: 160.9,134.3,118.8,115.4,113.3(\mathrm{t}, J=240 \mathrm{~Hz})$, $105.7,67.3(\mathrm{t}, J=30 \mathrm{~Hz}$ ); HRMS (APCI) calcd for $\mathrm{C}_{9} \mathrm{H}_{8} \mathrm{~F}_{2} \mathrm{NO}[\mathrm{M}+\mathrm{H}]^{+}: 184.0568$, found 184.0572 .

1-(2,2-Difluoroethoxy)-4-(trifluoromethyl)benzene (3j): Colorless liquid. ${ }^{1} \mathrm{H}$ NMR (400 MHz, $\left.\mathrm{CDCl}_{3}\right) \delta: 7.58(\mathrm{~d}$, $J=8.6 \mathrm{~Hz}, 2 \mathrm{H}), 6.99$ (d, $J=8.6 \mathrm{~Hz}, 2 \mathrm{H}), 6.11$ (tt, $J=54.8$, $4.0 \mathrm{~Hz}, 1 \mathrm{H}), 4.22(\mathrm{td}, J=12.8,4.0 \mathrm{~Hz}, 2 \mathrm{H}) ;{ }^{13} \mathrm{C}$ NMR $(100$ $\left.\mathrm{MHz}, \mathrm{CDCl}_{3}\right) \delta: 160.2,127.3(\mathrm{q}, J=4 \mathrm{~Hz}), 124.4$ (q, $J=$ $33 \mathrm{~Hz}), 124.4$ (q, $J=70 \mathrm{~Hz}), 114.8,113.5$ (t, $J=240 \mathrm{~Hz})$, $67.4\left(\mathrm{t}, J=29 \mathrm{~Hz}\right.$ ); HRMS (APCI) calcd for $\mathrm{C}_{9} \mathrm{H}_{8} \mathrm{~F}_{5} \mathrm{O}$ : 226.0412 , found 226.0420 .

1-(2,2-Difluoroethoxy)-4-methoxybenzene (3k): Colorless liquid. ${ }^{1} \mathrm{H}$ NMR $\left(400 \mathrm{MHz}, \mathrm{CDCl}_{3}\right) \delta: 6.89 \sim 6.80(\mathrm{~m}$, $4 \mathrm{H}), 6.05$ (tt, $J=55.2,4.0 \mathrm{~Hz}, 2 \mathrm{H}), 4.12(\mathrm{td}, J=13.2,4.0$ $\mathrm{Hz}, 2 \mathrm{H}), 3.77$ (d, $J=2.5 \mathrm{~Hz}, 3 \mathrm{H}) ;{ }^{13} \mathrm{C}$ NMR $(100 \mathrm{MHz}$, $\left.\mathrm{CDCl}_{3}\right) \delta: 154.9,152.1,132.4,116.0,114.9,114.0(\mathrm{t}, J=$ $240 \mathrm{~Hz}), 68.4$ (t, $J=30 \mathrm{~Hz}$ ), 55.8; HRMS (APCI) calcd for $\mathrm{C}_{9} \mathrm{H}_{11} \mathrm{~F}_{2} \mathrm{O}_{2}[\mathrm{M}+\mathrm{H}]^{+}:$189.0722, found 189.0731.

4-(2,2-Difluoroethoxy)- $N, N$-dimethylbenzenamine (3I): Purple black solid, m.p. 59 61 ${ }^{\circ} \mathrm{C} ;{ }^{1} \mathrm{H}$ NMR $(400 \mathrm{MHz}$, $\left.\mathrm{CDCl}_{3}\right) \delta: 6.85 \sim 6.76(\mathrm{~m}, 2 \mathrm{H}), 6.71(\mathrm{~d}, J=7.6 \mathrm{~Hz}, 2 \mathrm{H})$, $5.98(\mathrm{tt}, J=55.2,4.0 \mathrm{~Hz}, 1 \mathrm{H}), 4.05(\mathrm{td}, J=13.2,4.0 \mathrm{~Hz}$, 2H), $2.82(\mathrm{~s}, 6 \mathrm{H}) ;{ }^{13} \mathrm{C} \mathrm{NMR}\left(100 \mathrm{MHz}, \mathrm{CDCl}_{3}\right) \delta: 116.1$, $115.0,114.1(\mathrm{t}, J=240 \mathrm{~Hz}), 111.7,100.2,68.8(\mathrm{t}, J=30$ $\mathrm{Hz}$ ), 41.9; HRMS (APCI) calcd for $\mathrm{C}_{10} \mathrm{H}_{14} \mathrm{~F}_{2} \mathrm{NO}[\mathrm{M}+\mathrm{H}]^{+}$: 202.1038 , found 202.1045 .

4-(2,2-Difluoroethoxy)-1,2-dimethoxybenzene (3m): Pale yellow liquid. ${ }^{1} \mathrm{H}$ NMR $\left(400 \mathrm{MHz}, \mathrm{CDCl}_{3}\right) \delta: 6.76(\mathrm{t}$, $J=8.9 \mathrm{~Hz}, 1 \mathrm{H}), 6.55(\mathrm{~d}, J=2.8 \mathrm{~Hz}, 1 \mathrm{H}), 6.39$ (dd, $J=8.7$, $2.8 \mathrm{~Hz}, 1 \mathrm{H}), 6.06(\mathrm{tt}, J=55.2,4.1 \mathrm{~Hz}, 1 \mathrm{H}), 4.13(\mathrm{td}, J=$ 13.2, 4.1 Hz, 2H), $3.84(\mathrm{~d}, J=6.5 \mathrm{~Hz}, 6 \mathrm{H}) ;{ }^{13} \mathrm{C} \mathrm{NMR}(100$ $\left.\mathrm{MHz} \mathrm{CDCl}_{3}\right) \delta: 152.4,150.1,144.5,113.8(\mathrm{t}, J=240 \mathrm{~Hz})$, 111.8, 104.1, 101.3, $68.0(\mathrm{t}, J=30 \mathrm{~Hz}), 56.4,55.9,25.7$; HRMS (APCI) calcd for $\mathrm{C}_{10} \mathrm{H}_{13} \mathrm{~F}_{2} \mathrm{O}[\mathrm{M}+\mathrm{H}]^{+}:$219.0827, found 219.0833 .

1-(2,2-Difluoroethoxy)-3,5-dimethylbenzene (3n): Colorless liquid. ${ }^{1} \mathrm{H}$ NMR $\left(400 \mathrm{MHz}, \mathrm{CDCl}_{3}\right) \delta: 6.58(\mathrm{~s}, 1 \mathrm{H})$, $6.46(\mathrm{~s}, 2 \mathrm{H}), 5.98(\mathrm{tt}, J=55.2,4.4 \mathrm{~Hz}, 1 \mathrm{H}), 4.06(\mathrm{td}, J=$ 13.2, $4.8 \mathrm{~Hz}, 2 \mathrm{H}), 2.21(\mathrm{~s}, 6 \mathrm{H}) ;{ }^{13} \mathrm{C} \mathrm{NMR}(100 \mathrm{MHz}$, $\left.\mathrm{CDCl}_{3}\right) \delta: 157.9,139.7,123.8,114.0(\mathrm{t}, J=240 \mathrm{~Hz}), 112.5$, $67.4(\mathrm{t}, J=29 \mathrm{~Hz}), 21.5$; HRMS (APCI) calcd for $\mathrm{C}_{10} \mathrm{H}_{13} \mathrm{~F}_{2} \mathrm{O}[\mathrm{M}+\mathrm{H}]^{+}: 187.0929$, found 187.0922.

1-(2,2-Difluoroethoxy)-3,5-dimethoxybenzene (3o): Co- 
lorless liquid. ${ }^{1} \mathrm{H}$ NMR $\left(400 \mathrm{MHz}, \mathrm{CDCl}_{3}\right) \delta: 6.13 \sim 6.14$ $(\mathrm{m}, 1 \mathrm{H}), 6.08(\mathrm{~m}, 2 \mathrm{H}), 6.07$ (tt, $J=55.2,4.4 \mathrm{~Hz}, 1 \mathrm{H}), 4.13$ $(\mathrm{td}, J=13.2,4.0 \mathrm{~Hz}, 2 \mathrm{H}), 3.77(\mathrm{~s}, 6 \mathrm{H}) ;{ }^{13} \mathrm{C}$ NMR $(100$ $\left.\mathrm{MHz}, \mathrm{CDCl}_{3}\right) \delta: 161.7,159.7,113.7$ (t, $\left.J=240 \mathrm{~Hz}\right), 94.1$, 93.6, $67.3(\mathrm{t}, J=30 \mathrm{~Hz}), 55.5$; HRMS (ESI) calcd for $\mathrm{C}_{16} \mathrm{H}_{25} \mathrm{~F}_{2} \mathrm{O}[\mathrm{M}+\mathrm{H}]^{+}: 219.0827$, found 219.0824.

1,3-Di-tert-butyl-5-(2,2-Difluoroethoxy)benzene (3p): Colorless liquid. ${ }^{1} \mathrm{H}$ NMR $\left(400 \mathrm{MHz}, \mathrm{CDCl}_{3}\right) \delta$ : $7.09(\mathrm{t}$, $J=1.4 \mathrm{~Hz}, 1 \mathrm{H}), 6.77(\mathrm{~d}, J=1.5 \mathrm{~Hz}, 2 \mathrm{H}), 6.09$ (tt, $J=55.2$, $4.0 \mathrm{~Hz}, 1 \mathrm{H}), 4.18(\mathrm{td}, J=13.2,4.4 \mathrm{~Hz}, 2 \mathrm{H}), 1.30(\mathrm{~d}, J=7.8$ $\mathrm{Hz}, 18 \mathrm{H}) ;{ }^{13} \mathrm{C}$ NMR $\left(100 \mathrm{MHz}, \mathrm{CDCl}_{3}\right) \delta: 157.5,152.8$, 116.4, $114.1(\mathrm{t}, J=240 \mathrm{~Hz}), 109.1,67.5(\mathrm{t}, J=30 \mathrm{~Hz})$, 35.2, 31.6; HRMS (APCI) calcd for $\mathrm{C}_{16} \mathrm{H}_{25} \mathrm{~F}_{2} \mathrm{O}[\mathrm{M}+\mathrm{H}]^{+}$: 271.1868 , found 271.1875 .

1-(2,2-Difluoroethoxy)-3,5-bis(trifluoromethyl)benzene (3q): Colorless liquid. ${ }^{1} \mathrm{H} \mathrm{NMR}\left(400 \mathrm{MHz}, \mathrm{CDCl}_{3}\right) \delta$ : 7.55 (s, 1H), 7.35 (s, 2H), $6.13(\mathrm{tt}, J=54.8,4.0 \mathrm{~Hz}, 1 \mathrm{H}), 4.27$ (tt, $J=12.8,4.0 \mathrm{~Hz}, 2 \mathrm{H}) ;{ }^{13} \mathrm{C}$ NMR $\left(100 \mathrm{MHz}, \mathrm{CDCl}_{3}\right) \delta$ : 158.4, 133.4 (q, $J=33 \mathrm{~Hz}), 123.1(\mathrm{q}, J=271 \mathrm{~Hz}), 115.8$ $(\mathrm{q}, J=4 \mathrm{~Hz}), 115.2,113.1(\mathrm{t}, J=240 \mathrm{~Hz}), 67.8(\mathrm{t}, J=30$ $\mathrm{Hz}$ ); HRMS (APCI) calcd for $\mathrm{C}_{10} \mathrm{H}_{6} \mathrm{~F}_{8} \mathrm{O}: 294.0285$, found 294.0292 .

5-(2,2-Difluoroethoxy)-1,2,3-trimethoxybenzene (3r): Colorless liquid. ${ }^{1} \mathrm{H}$ NMR $\left(400 \mathrm{MHz}, \mathrm{CDCl}_{3}\right) \delta: 6.21(\mathrm{t}$, $J=4.0 \mathrm{~Hz}, 0.25 \mathrm{H}), 6.17(\mathrm{~s}, 2 \mathrm{H}), 6.07$ (t, $J=4.2 \mathrm{~Hz}, 0.5 \mathrm{H})$, $5.94(\mathrm{t}, J=4.2 \mathrm{~Hz}, 0.25 \mathrm{H}), 4.16(\mathrm{td}, J=13.2,4.0 \mathrm{~Hz}, 2 \mathrm{H})$, $3.85(\mathrm{~s}, 6 \mathrm{H}), 3.79(\mathrm{~s}, 3 \mathrm{H}) ;{ }^{13} \mathrm{C}$ NMR $\left(100 \mathrm{MHz}, \mathrm{CDCl}_{3}\right) \delta$ : $154.5,154.0,133.4,113.8(\mathrm{t}, J=240 \mathrm{~Hz}), 92.8,68.0$ (t, $J=29 \mathrm{~Hz}$ ), 61.2, 56.3; HRMS (APCI) calcd for $\mathrm{C}_{11} \mathrm{H}_{15} \mathrm{~F}_{2} \mathrm{O}_{4}[\mathrm{M}+\mathrm{H}]^{+}:$249.0933, found 249.0940.

1-(2-(2,2-Difluoroethoxy)phenyl)ethanone (3s): Pale yellow solid, m.p. $76 \sim 78{ }^{\circ} \mathrm{C} ;{ }^{1} \mathrm{H}$ NMR $(400 \mathrm{MHz}$, $\left.\mathrm{CDCl}_{3}\right) \delta: 7.77(\mathrm{dd}, J=7.6,1.6 \mathrm{~Hz}, 1 \mathrm{H}), 7.53 \sim 7.43(\mathrm{~m}$, $1 \mathrm{H}), 7.09$ (t, $J=8.0 \mathrm{~Hz}, 1 \mathrm{H}), 6.91(\mathrm{~d}, J=8.0 \mathrm{~Hz}, 1 \mathrm{H}), 6.16$ (tt, $J=54.8,4.0 \mathrm{~Hz}, 1 \mathrm{H}), 4.29(\mathrm{td}, J=13.2,4.0 \mathrm{~Hz}, 2 \mathrm{H})$, $2.63(\mathrm{~s}, 3 \mathrm{H}) ;{ }^{13} \mathrm{C} \mathrm{NMR}\left(100 \mathrm{MHz}, \mathrm{CDCl}_{3}\right) \delta: 199.3,156.8$, 133.8, 130.9, 128.9, 122.2, 113.3 (t, $J=240 \mathrm{~Hz}), 112.4$, $67.6(\mathrm{t}, J=29 \mathrm{~Hz}$ ), 32.0; HRMS (APCI) calcd for $\mathrm{C}_{10} \mathrm{H}_{11} \mathrm{~F}_{2} \mathrm{O}_{2}[\mathrm{M}+\mathrm{H}]^{+}:$201.0722, found 201.0726.

2-(2,2-Difluoroethoxy)biphenyl (3t): Yellow liquid. ${ }^{1} \mathrm{H}$ NMR $\left(400 \mathrm{MHz}, \mathrm{CDCl}_{3}\right) \delta: 7.48 \sim 7.41(\mathrm{~m}, 2 \mathrm{H}), 7.37 \sim$ $7.21(\mathrm{~m}, 5 \mathrm{H}), 7.04(\mathrm{td}, J=7.6,0.8 \mathrm{~Hz}, 1 \mathrm{H}), 6.89(\mathrm{~d}, J=8.1$ $\mathrm{Hz}, 1 \mathrm{H}), 5.88$ (tt, $J=55.3,4.2 \mathrm{~Hz}, 1 \mathrm{H}), 4.05(\mathrm{td}, J=12.8$, $4.0 \mathrm{~Hz}, 2 \mathrm{H}) ;{ }^{13} \mathrm{C}$ NMR $\left(100 \mathrm{MHz}, \mathrm{CDCl}_{3}\right) \delta: 154.9,138.0$, $131.8,131.4,129.6,128.8,128.2,127.3,122.7,113.9(\mathrm{t}$, $J=240 \mathrm{~Hz}), 113.6,68.4(\mathrm{t}, J=30 \mathrm{~Hz}), 31.8,29.8,1.9$; HRMS (APCI) calcd for $\mathrm{C}_{14} \mathrm{H}_{13} \mathrm{~F}_{2} \mathrm{O}[\mathrm{M}+\mathrm{H}]^{+}: 235.0929$, found 235.0935 .

1-(2,2-Difluoroethoxy)-2-fluorobenzene (3u): Light yellow liquid. ${ }^{1} \mathrm{H}$ NMR (400 MHz, $\left.\mathrm{CDCl}_{3}\right) \delta: 6.97 \sim 7.05$ $(\mathrm{m}, 2 \mathrm{H}), 6.89 \sim 6.93(\mathrm{~m}, 2 \mathrm{H}), 6.03(\mathrm{tt}, J=56.0,4.0 \mathrm{~Hz}$, $1 \mathrm{H}), 4.05(\mathrm{td}, J=12.0,4.0 \mathrm{~Hz}, 2 \mathrm{H}) ;{ }^{13} \mathrm{C} \mathrm{NMR}(100 \mathrm{MHz}$, $\left.\mathrm{CDCl}_{3}\right) \delta: 153.0(\mathrm{~d}, J=245 \mathrm{~Hz}), 145.8(\mathrm{~d}, J=10 \mathrm{~Hz})$, 124.5, 124.4, $123.0(\mathrm{~d}, J=7 \mathrm{~Hz}), 116.7(\mathrm{t}, J=18 \mathrm{~Hz})$, $113.6(\mathrm{t}, J=240 \mathrm{~Hz}), 69.1(\mathrm{t}, J=29 \mathrm{~Hz})$; MS (EI) $\mathrm{m} / \mathrm{z}$ : $176,157,125,111,95,81,65$.

3-(2,2-Difluoroethoxy)pyridine (3v): Light yellow liq- uid. ${ }^{1} \mathrm{H}$ NMR (400 MHz, $\left.\mathrm{CDCl}_{3}\right) \delta: 8.35(\mathrm{~s}, 1 \mathrm{H}), 8.30(\mathrm{~m}$, $1 \mathrm{H}), 7.23 \sim 7.28(\mathrm{~m}, 2 \mathrm{H}), 6.11(\mathrm{tt}, J=56.0,4.0 \mathrm{~Hz}, 1 \mathrm{H})$, $4.24(\mathrm{td}, J=16.0,4.0 \mathrm{~Hz}, 2 \mathrm{H}) ;{ }^{13} \mathrm{C}$ NMR $(100 \mathrm{MHz}$, $\left.\mathrm{CDCl}_{3}\right) \delta: 154.0,143.4,137.9,124.0,121.5,113.3(\mathrm{t}, J=$ $240 \mathrm{~Hz}), 67.4$ (t, $J=30 \mathrm{~Hz}$ ); MS (EI) $m / z: 159,108,95,91$, 78, 73. HRMS (ESI) calcd for $\mathrm{C}_{7} \mathrm{H}_{8} \mathrm{~F}_{2} \mathrm{NO}[\mathrm{M}+\mathrm{H}]^{+}$: 160.0568 , found 160.0568 .

1-(2,2-Difluoroethoxy)-3-nitrobenzene $\quad \mathbf{( 5 c )}$ : Yellow solid, m.p. $41 \sim 43{ }^{\circ} \mathrm{C},{ }^{1} \mathrm{H}$ NMR $\left(400 \mathrm{MHz}, \mathrm{CDCl}_{3}\right) \delta$ : $7.91(\mathrm{dd}, J=8.0,1.8 \mathrm{~Hz}, 1 \mathrm{H}), 7.76(\mathrm{t}, J=2.2 \mathrm{~Hz}, 1 \mathrm{H}), 7.49$ (t, $J=8.2 \mathrm{~Hz}, 1 \mathrm{H}), 7.28(\mathrm{dd}, J=8.4,1.6 \mathrm{~Hz}, 1 \mathrm{H}), 6.13$ (tt, $J=54.8,4.0 \mathrm{~Hz}, 1 \mathrm{H}), 4.28(\mathrm{td}, J=12.8,4.0 \mathrm{~Hz}, 2 \mathrm{H})$; ${ }^{13} \mathrm{C}$ NMR $\left(100 \mathrm{MHz}, \mathrm{CDCl}_{3}\right) \delta: 158.3,149.4,130.5,121.8$, 117.2, $113.3(\mathrm{t}, J=240 \mathrm{~Hz}), 109.2,67.8(\mathrm{t}, J=30 \mathrm{~Hz})$; HRMS (APCI) calcd for $\mathrm{C}_{8} \mathrm{H}_{8} \mathrm{~F}_{2} \mathrm{NO}_{3}[\mathrm{M}+\mathrm{H}]^{+}:$204.0467, found 204.0472 .

1-(2,2-Difluoroethoxy)-2-nitrobenzene (5f): Yellow solid, m.p. $50 \sim 52{ }^{\circ} \mathrm{C} ;{ }^{1} \mathrm{H}$ NMR $\left(400 \mathrm{MHz}, \mathrm{CDCl}_{3}\right) \delta$ : $7.87(\mathrm{dd}, J=8.4,1.6 \mathrm{~Hz}, 1 \mathrm{H}), 7.57(\mathrm{td}, J=8.4,1.6 \mathrm{~Hz}$, $1 \mathrm{H}), 7.18 \sim 7.07(\mathrm{~m}, 2 \mathrm{H}), 6.15(\mathrm{tt}, J=54.8,4.0 \mathrm{~Hz}, 1 \mathrm{H})$, $4.32(\mathrm{td}, J=12.8,4.4 \mathrm{~Hz}, 2 \mathrm{H}) ;{ }^{13} \mathrm{C} \mathrm{NMR}(100 \mathrm{MHz}$, $\left.\mathrm{CDCl}_{3}\right) \delta: 151.2,140.7,134.3,126.0,122.4,115.8,113.3$ (t, $J=240 \mathrm{~Hz}), 69.2(\mathrm{t}, J=30 \mathrm{~Hz}$ ); HRMS (APCI) calcd for $\mathrm{C}_{8} \mathrm{H}_{8} \mathrm{~F}_{2} \mathrm{NO}_{3}[\mathrm{M}+\mathrm{H}]^{+}:$204.0467, found 204.0476.

2-(2,2-Difluoroethoxy)benzenamine (5h): Black oil. ${ }^{1} \mathrm{H}$ NMR $\left(400 \mathrm{MHz}, \mathrm{CDCl}_{3}\right) \delta: 6.77(\mathrm{td}, J=8.0,1.6 \mathrm{~Hz}, 1 \mathrm{H})$, $6.71 \sim 6.57(\mathrm{~m}, 3 \mathrm{H}), 6.00(\mathrm{tt}, J=55.2,4.4 \mathrm{~Hz}, 1 \mathrm{H}), 4.10$ $(\mathrm{td}, J=13.2,4.4 \mathrm{~Hz}, 2 \mathrm{H}), 3.72(\mathrm{~s}, 2 \mathrm{H}) ;{ }^{13} \mathrm{C}$ NMR $(100$ $\left.\mathrm{MHz}, \mathrm{CDCl}_{3}\right) \delta: 145.4,136.8,122.9,120.0,118.5,115.8$, $113.8(\mathrm{t}, J=240 \mathrm{~Hz}), 112.6,68.0(\mathrm{t}, J=29 \mathrm{~Hz})$; HRMS (APCI) calcd for $\mathrm{C}_{8} \mathrm{H}_{10} \mathrm{~F}_{2} \mathrm{NO}[\mathrm{M}+\mathrm{H}]^{+}: 174.0725$, found 174.0730 .

2-(2,2-Difluoroethoxy)benzonitrile (5i): Colorless liquid. ${ }^{1} \mathrm{H}$ NMR $\left(400 \mathrm{MHz}, \mathrm{CDCl}_{3}\right) \delta: 7.49(\mathrm{dd}, J=15.6,7.6$ $\mathrm{Hz}, 2 \mathrm{H}), 7.02(\mathrm{dd}, J=7.6,0.8 \mathrm{~Hz}, 1 \mathrm{H}), 6.91(\mathrm{~d}, J=8.3 \mathrm{~Hz}$, $1 \mathrm{H}), 6.08$ (tt, $J=54.8,4.0 \mathrm{~Hz}, 1 \mathrm{H}), 4.23(\mathrm{td}, J=12.8,4.4$ $\mathrm{Hz}, 2 \mathrm{H}) ;{ }^{13} \mathrm{C} \mathrm{NMR}\left(100 \mathrm{MHz}, \mathrm{CDCl}_{3}\right) \delta: 159.2,134.6$, $134.1,122.3,115.8,113.3(\mathrm{t}, J=240 \mathrm{~Hz}), 112.7,102.7$, $68.0(\mathrm{t}, J=30 \mathrm{~Hz})$; HRMS (APCI) calcd for $\mathrm{C}_{9} \mathrm{H}_{8} \mathrm{~F}_{2} \mathrm{NO}$ $[\mathrm{M}+\mathrm{H}]^{+}: 184.0568$, found 184.0572 .

1-(2,2-Difluoroethoxy)-4-nitrobenzene (5j): Light yellow solid, m.p. 87 $\sim 88{ }^{\circ} \mathrm{C} ;{ }^{1} \mathrm{H}$ NMR $\left(400 \mathrm{MHz}, \mathrm{CDCl}_{3}\right) \delta$ : $788(\mathrm{~d}, J=1.6 \mathrm{~Hz}, 1 \mathrm{H}), 7.86 \sim 7.58(\mathrm{~m}, 1 \mathrm{H}), 7.18 \sim 7.09$ $(\mathrm{m}, 2 \mathrm{H}), 6.14(\mathrm{tt}, J=54.8,4.0 \mathrm{~Hz}, 1 \mathrm{H}), 4.29(\mathrm{td}, J=12.8$, $4.0 \mathrm{~Hz}, 2 \mathrm{H}) ;{ }^{13} \mathrm{C} \mathrm{NMR}\left(100 \mathrm{MHz}, \mathrm{CDCl}_{3}\right) \delta: 162.5,142.6$, 126.2, 114.8, $113.2(\mathrm{t}, J=240 \mathrm{~Hz}), 67.6(\mathrm{t}, J=30 \mathrm{~Hz})$; HRMS (APCl) calcd for $\mathrm{C}_{8} \mathrm{H}_{8} \mathrm{~F}_{2} \mathrm{NO}_{3}[\mathrm{M}+\mathrm{H}]^{+}$204.0467, found 204.0475 .

1-(2,2-Difluoroethoxy)naphthalene (5k): Brown liquid. ${ }^{1} \mathrm{H}$ NMR $\left(400 \mathrm{MHz}, \mathrm{CDCl}_{3}\right) \delta: 8.22 \sim 8.14(\mathrm{~m}, 1 \mathrm{H}), 7.74$ $(\mathrm{dd}, J=5.6,2.4 \mathrm{~Hz}, 1 \mathrm{H}), 7.47 \sim 7.38(\mathrm{~m}, 3 \mathrm{H}), 7.29(\mathrm{t}, J=$ $8.0 \mathrm{~Hz}, 1 \mathrm{H}), 6.72(\mathrm{~d}, J=7.6 \mathrm{~Hz}, 1 \mathrm{H}), 6.16(\mathrm{tt}, J=55.2,4.0$ $\mathrm{Hz}, 1 \mathrm{H}), 4.28(\mathrm{td}, J=12.8,4.0 \mathrm{~Hz}, 2 \mathrm{H}) ;{ }^{13} \mathrm{C}$ NMR $(100$ $\left.\mathrm{MHz}, \mathrm{CDCl}_{3}\right) \delta: 153.6,134.7,127.7,126.9,125.8,125.7$, 125.6, 121.9, 121.8, $113.9(\mathrm{t}, J=240 \mathrm{~Hz}), 105.3,67.7(\mathrm{t}$, $J=30 \mathrm{~Hz}$ ); HRMS (APCI) calcd for $\mathrm{C}_{12} \mathrm{H}_{11} \mathrm{~F}_{2} \mathrm{O}[\mathrm{M}+\mathrm{H}]^{+}$: 209.0772, found 209.0780. 
Supporting Information NMR spectra for compounds, ESI-MS analysis of reaction intermediates, and DFT studies are available free of charge in the Supporting Information via the Internet at http://sioc-journal.cn/.

\section{References}

[1] (a) Nakajima, T.; Groult, H. Fluorinated Materials for Energy Conversion, Elsevier, London, 2005.

(b) Wong, S.; Ma, H.; Jen, A. K. Y.; Barto, R.; Frank, C. W. Macromolecules $\mathbf{2 0 0 3}, 36,8001$.

(c) Wong, S.; Ma, H.; Jen, A. K. Y.; Barto, R.; Frank, C. W. Macromolecules 2004, 37, 5578 .

[2] (a) Tressaud, A.; Haufe, G. Fluorine and Health: Molecular Imaging, Biomedical Materials and Pharmaceuticals, Elsevier, London, 2008.

(b) Ojima, I. Fluorine in Medicinal Chemistry and Chemical Biology, Wiley, Hoboken, 2009.

(c) Xu, X. H.; Matsuzaki, K.; Shibata, N. Chem. Rev. 2015, 115, 731.

[3] Jeschke, P. ChemBioChem 2004, 5, 570.

[4] (a) Banks, R. E.; Smart, B. E.; Tatlow, J. C. Organofluorine Chemistry, Principles and Commercial Applications, Plenum, New York, 1994.

(b) Purser, S.; Moore, P. R.; Swallow, S.; Gouverneur, V. Chem. Soc. Rev. 2008, 37, 320 .

(c) Wang, J.; Sanchez-Rosello, M.; Acena, J. L.; del Pozo, C.; Sorochinsky, A. E.; Fustero, S.; Soloshonok, V. A.; Liu, H. Chem. Rev. 2014, 114, 2432.

[5] (a) Gao, X.; Cheng, R.; Xiao, Y. L.; Wan, X. L.; Zhang, X. G. Chem 2019, 5, 2987.

(b) Begue, J. P.; Bonnet-Delpon, D. J. Fluorine Chem. 2006, 127, 992.

(c) Cerqueira, N. M. F. S. A.; Fernandes, P. A.; Ramos, M. J. Chemistry 2007, 13, 8507.

(d) Teague, S. J. Drug Discovery Today 2011, 16, 398.

(e) Cheng, J. J.; Giguere, P. M.; Onajole, O. K.; Lv, W.; Gaisin, A.; Gunosewoyo, H.; Schmerberg, C.; Pogorelov, V. M.; Rodriguiz, R. M.; Vistoli, G.; Wetsel, W. C.; Roth, B. L.; Kozikowski, A. P. J. Med. Chem. 2015, 58, 1992

(f) Johan, B. WO 2010132016, 2010.

(g) Gregory, B. M. WO 02072528, 2002.

(h) Gernert, D. L.; Ajamie, R.; Ardecky, R. A.; Bell, M. G.; Leibowitz, M. D.; Mais, D. A.; Mapes, C. M.; Michellys, P. Y.; Rungta, D.; Reifel-Miller, A.; Tyhonas, J. S.; Yumibe, N.; Grese, T. A. Bioorg. Med. Chem. Lett. 2003, 13, 3191.

(i) Ulrich, K. WO 2005085203, 2005.

(j) Ulrich, K. WO $2006092417,2006$.

[6] (a) Irrupe, Jr. J.; Casas, J.; Messeguer, A. Bioorg. Med. Chem. Lett. 1993, 3, 179.

(b) Thomas, W. J.; Ronald, S. T. J. Agric. Food Chem. 2005, 53, 7179 .

[7] (a) Schwan, G.; Asskar, G. B.; Hcfgen, N.; Kubicova, L.; Funke, U.; Egerland, U.; Zahn, M.; Nieber, K.; Scheunemann, M.; Strter, N.; Brust, P.; Briel, D. ChemMedChem 2014, 9, 1476.

(b) OShea, P. D.; Gauvreau, D.; Gosselin, F.; Hughes, G.; Nadeau, C.; Roy, A.; Scott Shultz, C. J. Org. Chem. 2009, 74, 4547.

(c) Kamal, A.; Pratap, T. B.; Ramana, K. V.; Ramana, A. V.; Babu, A. H. Tetrahedron Lett. 2002, 43, 7353.

(d) Camps, F.; Coll, J.; Messeguer, A.; Perics, M. A. Synthesis $1980,727$.

[8] Fuhrmann, E.; Talbiersky, J. Org. Process Res. Dev. 2005, 9, 206.

[9] (a) Zhang, H.; Ruiz-Castillo, P.; Buchwald, S. L. Org. Lett. 2018, 20, 1580 .

(b) Gowrisankar, S.; Sergeev, A. G.; Anbarasan, P.; Spannenberg, A.; Neumann, H.; Beller, M. J. Am. Chem. Soc. 2010, 132, 11592.

(c) Sawatzky, R. S.; Hargreaves, B. K. V.; Stradiotto, M. Eur. J.
Org. Chem. 2016, 2016, 2444.

(d) Dumrath, A.; Wu, X. F.; Neumann, H.; Spannenberg, A.; Jackstell, R.; Beller, M. Angew. Chem., Int. Chem. 2010, 49, 8988.

(e) Mann, G.; Incarvito, C.; Rheingold, A. L.; Hartwig, J. F. J. Am. Chem. Soc. 1999, 121, 3224.

[10] Rangarajan, T. M.; Singh, R.; Brahma, R.; Devi, K.; Pal Singh, R.; Singh, R. P.; Prasad, A. K. Chem.-Eur. J. 2014, 20, 14218.

[11] (a) Ma, D.; Zhang, Y.; Yao, J.; Wu, S.; Tao, F. J. Am. Chem. Soc. 1998, 120, 12459 .

(b) Goodbrand, H. B.; Hu, N. X. J. Org. Chem. 1999, 64, 670.

(c) Fagan, P. J.; Hauptman, E.; Shapiro, R.; Casalnuovo, A. J. Am. Chem. Soc. 2000, 122, 5043.

[12] (a) Suzuki, H.; Matuoka, T.; Ohtsuka, I.; Osuka, A. Synthesis 1985, 499.

(b) Sugata, H.; Tsubogo, T.; Kino, Y.; Uchiro, H. Tetrahedron Lett. 2017, 58, 1015.

[13] (a) Wolter, M.; Nordmann, G.; Job, E.; Buchwald, S. L. Org. Lett. $\mathbf{2 0 0 2}, 4,973$.

(b) Tlili, A.; Xia, N.; Monnier, F.; Taillefer, M. Angew. Chem., Int. Chem. 2009, 48, 8725.

(c) Chen, Z. X.; Jiang, Y. W.; Zhang, L.; Guo, Y. L.; Ma, D. W. J. Am. Chem. Soc. 2019, 141, 3541

(d) Cai, Q.; Zhou, W. Chin. J. Chem. 2020, 38, 879.

[14] (a) Huang, R. L.; Huang, Y. J.; Lin, X. X.; Rong, M.; Weng, Z. Angew. Chem., Int. Ed. 2015, 54, 5736.

(b) Vuluga, D.; Legros, J.; Crousse, B.; Bonnet-Delpon, D. Eur. J. Org. Chem. 2009, 3513.

[15] Niu, J. J.; Guo, P. R.; Kang, J. T.; Li, Z. G.; Xu, J. W.; Hu, S. J. J. Org. Chem. 2009, 74, 5075.

[16] (a) Qian, C.; Zhu, W.; Liu, J.; Wang, X.; Qiu, L. Chin. J. Org. Chem. 2019, 39, 1695 (in Chinese).

(钱存卫，朱文倩，刘俊龙，王雪敏，仇立干，有机化学，2019, 39, 1695.)

(b) Liu, X.; Chen, W.; Ni, B.; Chen, X.; Qian, C.; Ge, X. Chin. J. Org. Chem. 2018, 38, 1703 (in Chinese).

(刘学民，陈雯，倪邦庆，陈新志，钱超，葛新，有机化学，2018， 38,1703 .)

(c) Hu, X.; Yang, B.; Yao, W.; Wang, D. Chin. J. Org. Chem. 2018, 38, 3296 (in Chinese).

(胡昕宇, 杨伯斌, 姚玮, 王大伟, 有机化学, 2018, 38, 3296.)

[17] (a) Ye, D.; Huang, R.; Zhu, H.; Zou, L.; Wang, D. Org. Chem. Front. 2019, 6, 62 .

(b) Hu, W.; Zhang, Y.; Zhu, H.; Ye, D.; Wang, D. Green Chem. 2019, 21, 5345.

(c) Wang, D.; Zhao, K.; Xu, C.; Miao, H.; Ding, Y. ACS Catal. 2014, 4, 3910.

[18] (a) Johansson, C. C. C.; Colacot, T. J. Angew. Chem., Int. Ed. 2010, 49,676 .

(b) Cristau, H. J.; Cellier, P. P.; Spinddler, J. F.; Taillefer, M. Chem.Eur. J. 2004, 10, 5607.

(c) Xie, X.; Cai, G.; Ma, D. Org. Lett. 2005, 7, 4693.

(d) Xie, X.; Chen, Y.; Ma, D. J. Am. Chem. Soc. 2006, 128, 16050.

[19] (a) Yu, H. Z.; Jiang, Y. Y.; Fu, Y.; Liu, L. J. Am. Chem. Soc. 2010, 132, 18078.

(b) Ribas, X.; Guell, I. Pure Appl. Chem. 2014, 86, 345.

(c) Jones, G. O.; Liu, P.; Houk, K. N.; Buchwald, S. L. J. Am. Chem. Soc. 2010, 132, 6205.

(d) Casitas, A.; Ribas, X. Chem. Sci. 2013, 4, 2301.

(e) Sambiagio, C.; Marsden, S. P.; Blacker, A. J.; McGowan, P. C. Chem. Soc. Rev. 2014, 43, 3525.

(f) Lefevre, G.; Franc, G.; Tlili, A.; Adamo, C.; Taillefer, M.; Ciofini, I.; Jutand, A. Organometallics 2012, 31, 7694.

(g) Giri, R.; Brusoe, A.; Troshin, K.; Wang, J. Y.; Font, M.; Hartwig, J. F. J. Am. Chem. Soc. 2018, 140, 793.

(h) Tye, J. W.; Weng, Z.; Giri, R.; Hartwig, J. F. Angew. Chem., Int. Ed. 2010, 49, 2185.

(Li, L.; Fan, Y.) 